\title{
An Implementation Strategy for the Use of Chromogenic Media in the Rapid, Presumptive Identification of Candida Species
}

\author{
Heather J. Adam ${ }^{*}, 1$, Susan E. Richardson ${ }^{1,2}$, Margaret Roscoe ${ }^{2}$, Shahram Boroumandi ${ }^{3}$, \\ Marlene Gris ${ }^{2}$ and Yvonne C. W. Yau ${ }^{1,2}$ \\ ${ }^{I}$ Department of Laboratory Medicine and Pathobiology, Faculty of Medicine, University of Toronto, Toronto, Ontario, \\ Canada \\ ${ }^{2}$ Division of Microbiology, Paediatric Laboratory Medicine, Hospital for Sick Children, Toronto, Ontario, Canada \\ ${ }^{3}$ Department of Medical Microbiology, Faculty of Medicine, University of Manitoba, Winnipeg, Manitoba, Canada
}

\begin{abstract}
The purpose of this study was to devise an algorithm utilizing chromogenic media for the detection and presumptive identification of Candida species in a clinical microbiology laboratory. In order to select one chromogenic medium, a limited evaluation of 3 commercially available yeast chromogenic media: BBL CHROMagar Candida, BioRad CandiSelect 4, and Oxoid OCCA, was conducted with 12 C. albicans, 8 C. glabrata, 10 C. tropicalis, and 9 C. krusei. The presumptive species identification after 48 hours of incubation on BBL and Bio-Rad media were comparable, with more than $80 \%$ of the Candida species correctly identified, and both were superior to Oxoid. An algorithm based on use of chromogenic media for the rapid presumptive identification of C. albicans, C. tropicalis, C. krusei, and C. glabrata was developed for routine use in the microbiology laboratory. A presumptive identification is available within 24 to 48 hours utilizing this algorithm.
\end{abstract}

Keywords: Chromagar, chromogenic media, candida.

\section{INTRODUCTION}

Yeasts are a common cause of nosocomial infection in hospitalized patients. Candida species are reported as the fourth most commonly isolated pathogen from blood cultures in hospitalized patients $[1,2]$. The most common Candida species causing bloodstream infections include $C$. albicans, C. glabrata, C. tropicalis, and C. parapsilosis [3]. Rapid identification of these non-albicans species is critical to patient management as $C$. krusei and C. glabrata exhibit decreased susceptibility to fluconazole [4], which is frequently selected for empiric therapy.

Conventional yeast identification includes microscopic morphological identification and biochemical studies, which require technical expertise and may take three to four days. Chromogenic media have recently been developed to facilitate simple and rapid yeast identification for the more commonly isolated species. Current chromogenic yeast media isolate and differentiate $C$. albicans, $C$. glabrata, $C$. krusei, and C. tropicalis based on colonial morphology and color production [5-10]. The identification is considered presumptive, particularly for C. glabrata, C. krusei, and $C$. tropicalis, and requires confirmatory testing by standard yeast identification methods. The use of chromogenic media as a primary medium for Candida species detection from clinical specimens is supported by the high sensitivity and specificity reported in the current literature $[5,9,10]$. Chromogenic media are advantageous as they also enable the detection of mixed yeast cultures, which may not be detected

*Address correspondence to this author at the Clinical Microbiology, Health Sciences Centre, MS673-820 Sherbrook, St., Winnipeg, Manitoba, R3A1R9, Canada; Tel: (204) 787-8678; E-mail: hadam@hsc.mb.ca on standard media due to the similar colonial morphology of different yeast species [11].

Currently, there are only 3 commercially available chromogenic yeast media: BBL CHROMagar Candida (CHROMagar; Becton Dickinson Canada Inc.), Bio-Rad CandiSelect 4 (CandiSelect; Bio-Rad), and Oxoid OCCA (OCCA; Oxoid Ltd.). Comparative studies have been conducted with CHROMagar and OCCA [12] as well as CHROMagar and CandiSelect $[10,13]$; however, a comparison of all three media has not been reported previously. In order to select the best chromogenic medium for use in the routine identification of yeast in a clinical microbiology laboratory, a limited evaluation of the 3 commercially available chromogenic yeast media was conducted.

The purpose of this study was to devise an algorithm to optimize the use of chromogenic media for the detection and presumptive identification of Candida species in a clinical microbiology laboratory. New microbiological products are frequently developed and made available to laboratories; however, the best way to incorporate these products into current laboratory processes is not always evident. This study was designed to address this question for yeast chromogenic media.

\section{MATERIALS AND METHODOLOGY}

\section{Candida Strains}

Thirty-nine Candida strains including 12 C. albicans, 8 C. glabrata, 10 C. tropicalis, and 9 C. krusei were evaluated in this study. All strains were selected from clinical bloodstream Candida isolates from patients in Toronto, 
Table 1. Colonial Morphology and Color Development on BBL CHROMagar Candida, Bio-Rad CandiSelect 4, and Oxoid OCCA for the Presumptive Identification of $C$. albicans, $C$. glabrata, $C$. tropicalis, and $C$. krusei

\begin{tabular}{|c|c|c|c|c|}
\hline Media & C. albicans & C. glabrata & C. tropicalis & C. rrusei \\
\hline \hline BBL CHROMagar Candida & $\begin{array}{c}\text { Light to medium } \\
\text { green }\end{array}$ & Small pink & Dark blue to metallic blue & $\begin{array}{c}\text { Light mauve to mauve with } \\
\text { whitish border, rough }\end{array}$ \\
\hline Bio-Rad CandiSelect 4 & Pink to purple & $\begin{array}{c}\text { Pale turquoise centre with white } \\
\text { periphery }\end{array}$ & Intense turquoise & Turquoise-blue, rough \\
\hline Oxoid OCCA & Green & Beige/yellow-brown & Dark blue & Pink-brown, dry \\
\hline
\end{tabular}

which are stored in the microbiology laboratory at the Hospital for Sick Children, Toronto, Canada. Isolates were identified by conventional means, i.e. germ tube test, morphology on cornmeal tween 80 agar and API 20C AUX.

\section{Candida Identification on Chromogenic Media}

Each Candida strain was subcultured twice onto blood agar from the stock culture prior to inoculation of the 3 chromogenic media (CHROMagar, CandiSelect, and OCCA). Standard inocula were prepared such that $30-100$ colonies were inoculated onto each of the 3 chromogenic media. In accordance with the manufacturers' instructions, the CHROMagar and CandiSelect were incubated aerobically at $35^{\circ} \mathrm{C}$ and the OCCA was incubated aerobically at $30^{\circ} \mathrm{C}$. Color development and colonial morphology were evaluated daily for 2 days and a presumptive identification of each isolate was recorded. The color and colonial morphology used to identify the organisms is detailed in Table 1. The selection of isolates and the evaluation of colony color and morphology on the chromogenic media were conducted by separate individuals to complete the study in a blinded manner.

The identification of 3 chlamydospore negative $C$. albicans was confirmed by DNA sequencing. Sequencing was conducted using ITS3 and ITS4 fungal primers previously described by Chen et al. [14].

A retrospective review of all blood cultures and sterile site specimens from 2007 and 2008 that included a yeast workup was conducted to validate the developed algorithm.

\section{RESULTS AND DISCUSSION}

\section{Accuracy of the Chromogenic Media}

All 3 media are able to identify C. albicans, C. krusei, and $C$. tropicalis. CandiSelect is marketed with an additional indication for the presumptive identification of C. glabrata. Although CHROMagar and OCCA are not marketed with an indication for C. glabrata, C. glabrata strains have a characteristic appearance on both media that is different from the three species for which they have been validated (see Table 1). As such, we evaluated their utility in making a presumptive diagnosis of $C$. glabrata infection. After 24 hours, CHROMagar, CandiSelect, and OCCA correctly identified $36 \%, 49 \%$, and $3 \%$ of the Candida species, respectively. By 48 hours, $85 \%, 85 \%$, and $62 \%$ of the Candida species were correctly identified by CHROMagar, CandiSelect, and OCCA, respectively. The overall percent identification of Candida species provided by each media after 24 and 48 hours of incubation is presented in Fig. (1). As demonstrated in Fig. (2), CHROMagar and CandiSelect correctly identified all $C$. albicans and $C$. krusei by 48 hours whereas OCCA only identified $92 \%$ of the $C$. albicans and $56 \%$ of the $C$. krusei. At 48 hours, CHROMagar, CandiSelect, and OCCA identified $50 \%, 60 \%$, and $40 \%$ of the $C$. tropicalis. CHROMagar, CandiSelect, and OCCA correctly identified $88 \%, 75 \%$, and $50 \%$ of C. glabrata strains by 48 hours, respectively.

Three chlamydospore negative $C$. albicans were correctly identified as $C$. albicans on all three chromogenic media after 24 to 48 hours of incubation. Sequencing of the rRNA genes confirmed the $C$. albicans identification.

A small number of discordant identifications were observed on the three media. The discordant identifications were as follows: CHROMagar, $1 C$. tropicalis identified as C. glabrata; CandiSelect, 1 C. glabrata identified as $C$. tropicalis; OCCA, 1 C. albicans identified as C. tropicalis, 1 C. tropicalis identified as $C$. albicans, and 1 C. tropicalis identified as C. glabrata.

\section{Selection of a Chromogenic Medium for Routine Use}

The amount of time required for identification and the accuracy of the species identification were comparable for CHROMagar and CandiSelect. As shown in Fig. (1), CHROMagar (98\%) provided a greater percentage of correct or possible identifications at 48 hours than either CandiSelect $(85 \%)$ or OCCA $(80 \%)$. However, CHROMagar and CandiSelect were comparable in their ability to identify the 4 Candida species: C. albicans, C. glabrata, C. krusei, and C. tropicalis (Fig. 2). Similarly, Sendid et al. demonstrated that an equivalent proportion of $C$. albicans, C. glabrata, $C$. krusei, and $C$. tropicalis isolates was identified by CHROMagar and CandiSelect [13]. OCCA was found to be inferior to CHROMagar and CandiSelect at 24 and 48 hours with respect to the speed of the presumptive identification and the proportion of correct identifications, as previously reported [12]. A 72 hour incubation is recommended by the manufacturer of OCCA; however, we required a presumptive identification within 48 hours for the development of a rapid identification schema. Accordingly, the results after 72 hours of incubation were not considered in our study. The observation that CHROMagar was superior to OCCA supports the current literature [12].

Although CandiSelect is advantageous as it provides a presumptive identification for C. glabrata, the distinct color development of the four Candida species on CHROMagar was easier for the technologists to learn and may result in 


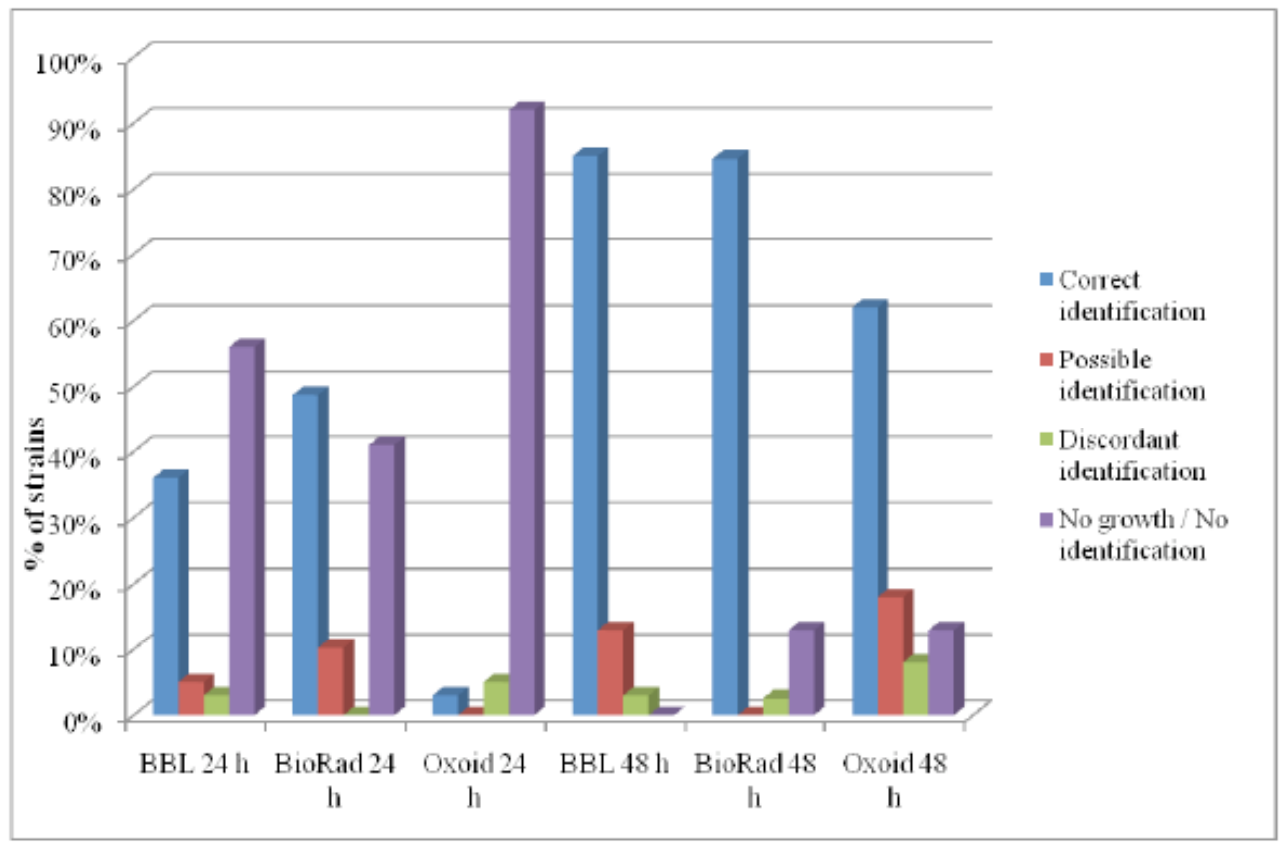

Fig. (1). Overall Percent Identification of Candida species Provided by BBL CHROMagar Candida, Bio-Rad CandiSelect 4, and Oxoid OCCA after 24 and 48 Hours of Incubation.

Correct identification, identification matches database; Possible identification, color development indicative, but inconclusive for identification (e.g. Mauve colony on CHROMagar indicative of $C$. krusei, but inconclusive without the white border); discordant identification, identification discordant with database; and no identification, no species-specific color development.

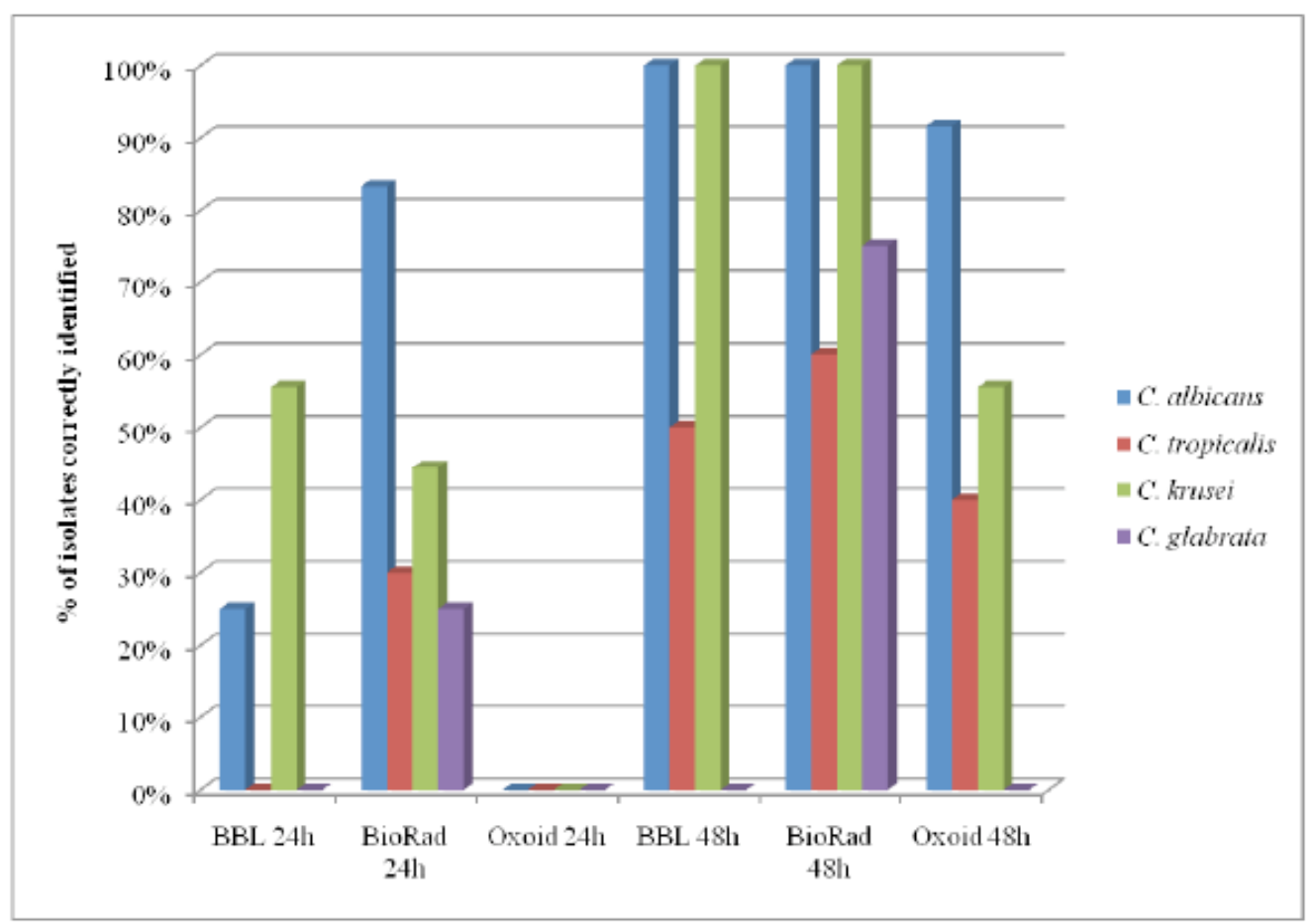

Fig. (2). Percent of $C$. albicans, $C$. glabrata, . krusei, and $C$. tropicalis Correctly Identified by BBL CHROMagar Candida, Bio-Rad CandiSelect 4, and Oxoid OCCA after 24 and 48 Hours of Incubation.

greater consistency of yeast identification. The rationale for the ultimate selection of CHROMagar in our laboratory included its demonstrated accuracy in this study, the wide acceptance of CHROMagar as the medium of choice based on favorable reports in the literature $[5,6,8,9,11,12]$, and the preference by the laboratory technologists due to its distinct color profile. 


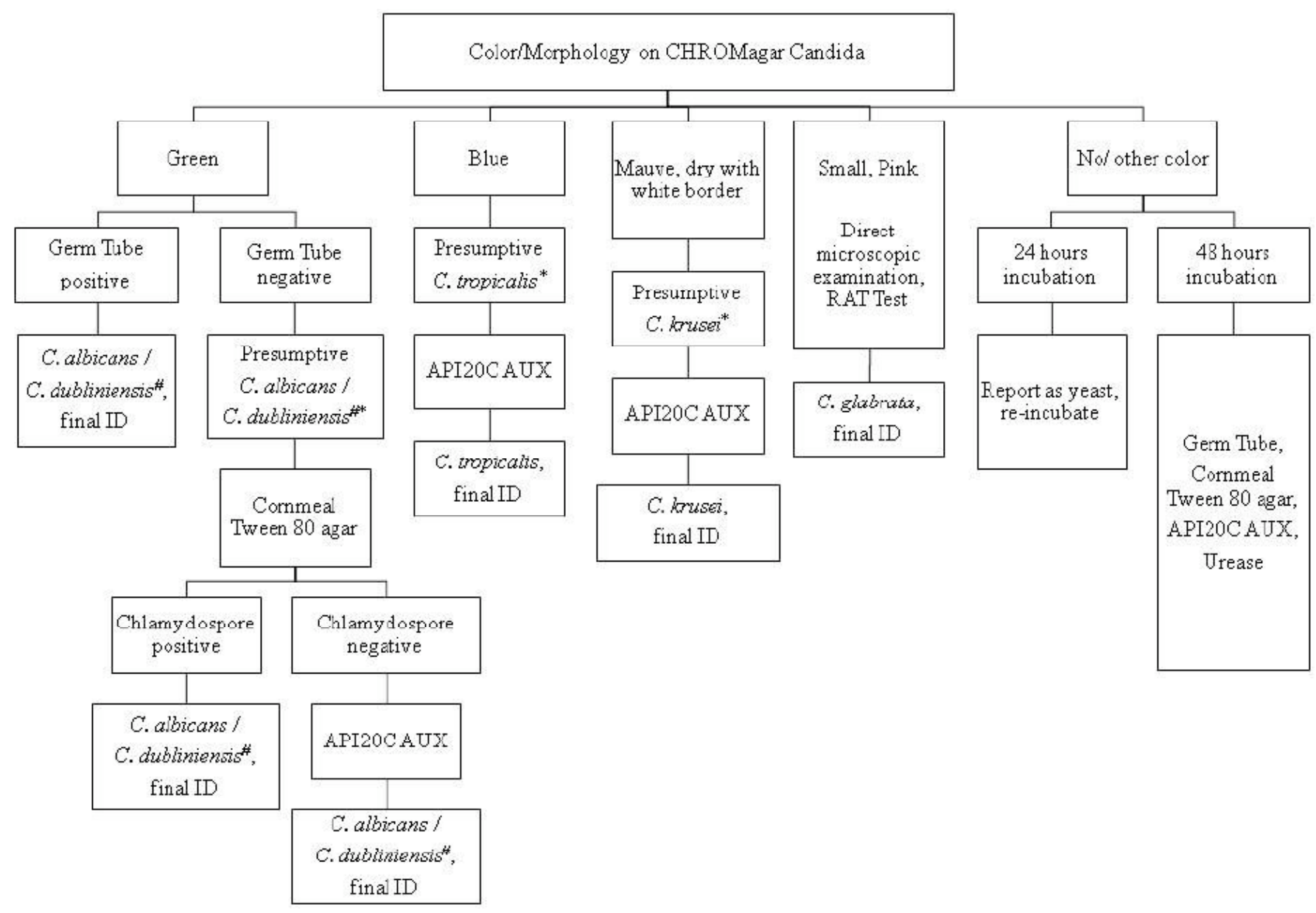

Fig. (3). Flowchart Detailing the Implementation of BBL CHROMagar Candida Media for Routine Yeast Identification.

\#, C. albicans cannot be distinguished from C. dubliniensis by these methods; *, At this point, an interim result can be issued i.e."Presumptive $C$. (

\section{Targeted Implementation of BBL CHROMagar Candida}

In order to utilize the CHROMagar medium in a costeffective manner in our laboratory, the medium is included in the routine investigation of specimens for which complete yeast identification is warranted. In particular, the medium is utilized as a primary isolation medium for sterile site cultures, including blood, CSF, sterile fluids (abdominal, joint, pericardial, peritoneal), and biopsies.

The identification schema developed for the implementation of CHROMagar for routine yeast identification from sterile sites is presented in Fig. (3). This schema enables the rapid presumptive identification of C. albicans, C. tropicalis, and $C$. krusei. As CHROMagar is not marketed for the presumptive identification of C. glabrata, a rapid confirmatory test is suggested rather than a presumptive identification based on colony colour. In our schema, a presumptive identification is made based on the color and colonial morphology observed on the chromogenic media as soon as it is possible, following 24 or 48 hours of incubation. At that point, an interim report is provided to the clinician with the presumptive yeast identification and a comment that confirmation will be forthcoming.
A final identification requires confirmatory testing. As per our schema, the additional tests differ based on the presumptive identification and include morphology on direct microscopic examination, morphology on cornmeal tween 80 agar, detection of urease, API 20C AUX results, and RAT (rapid assimilation of trehalose) test (REMEL Inc., Lenexa, KS). The identification of C. glabrata from CHROMagar with our schema relies on the presence of typical macroscopic morphology (small pink), typical microscopic morphology (small, circular yeast cells) [15], and a positive RAT test. The RAT test has been shown to be highly sensitive and specific in the confirmation of $C$. glabrata identification from chromogenic media [8]. The confirmatory tests for $C$. glabrata can be completed on the same day as the pink colonies are observed, which permits a rapid final identification on the same day that a presumptive identification would be available. Isolates with discordant presumptive identifications are subjected to additional testing: Cornmeal Tween 80 agar from presumptive $C$. tropicalis and C. krusei and Cornmeal Tween 80 agar and API20C AUX for presumptive C. glabrata.

C. dubliniensis cannot be definitively distinguished from C. albicans on chromogenic media [6]; thus, green colonies 
are reported as $C$. albicans/C. dubliniensis. Most Candida yeasts other than $C$. albicans, $C$. glabrata, $C$. krusei, and $C$. tropicalis appear to be shades of pink, lavender or ivory, which are distinguishable from the four yeasts for which a presumptive identification is possible. However, several uncommon strains of Candida, including $C$. inconspicua, $C$. firmetaria, and some strains of $C$. norvegensis and $C$. lipolytica, may have the rough colonial morphology, pink/purple hue and pale border characteristic of C. krusei, making them indistinguishable morphologically [6]. Thus, confirmation of the identify of $C$. krusei is required, in this case with an API 20 AUX.

The accuracy of the chromogenic media also enables it to be relied upon for the identification of repeat positive specimens. C. albicans/C. dubliniensis, C. glabrata, C. krusei or $C$. tropicalis that are re-isolated from the same site within two weeks of a complete identification are referred to the previous identification based on the color and colonial morphology observed on the chromogenic medium without further testing.

\section{POST-IMPLEMENTATION REVIEW OF CANDIDA SPP. IDENTIFICATIONS WITH THE RAPID, PRESUMPTIVE IDENTIFICATION ALGORITHM UTILIZING BBL CHROMAGAR CANDIDA MEDIA}

Since 2007, 102 blood culture and 26 sterile site specimens were processed with the yeast chromogenic media following the algorithm detailed in Fig. (3). There were 78 blood cultures from 21 patients and 21 sterile site isolates from 6 patients available for full analysis upon retrospective review. Seventy-one C. albicans, 7 C. glabrata, 1 C. krusei, and 19 C. parapsilosis were recovered from these specimens. The presumptive identifications provided by the yeast chromogenic media were confirmed for all isolates of $C$. glabrata, C. krusei and $98.6 \%$ of the $C$. albicans. One culture with $C$. albicans grew as 2 morphotypes; one morphotype originally grew as a white colony on the yeast chromogenic media. The average time to presumptive identification for C. albicans, C. glabrata, and C. krusei was 1.5 days (range: 1-4 days), 3 days (range: 2-4 days), and 1 day, respectively. The average time to final identification for C. albicans, C. glabrata, C. krusei, and C. parapsilosis was 2.3 days (range: 1-9 days), 3.7 days (range: 3-4 days), 5 days, and 4.4 days (range: 3-6 days), respectively.

\section{CONCLUSION}

Significant work evaluating the sensitivity and specificity of yeast chromogenic media has been reported previously [5, $6,8-10,12,13]$; however, none of them has suggested implementation schemes for routine use in hospital microbiology laboratories. This study is unique as it details a simple and practical method for incorporating chromogenic media into routine yeast identification from specimens for which complete yeast identification is warranted. In the retrospective review following the implementation of the algorithm, it was shown to provide accurate identifications to clinicians by at least one day earlier than the final identifications completed by traditional methods.
The ability to refer specimen identifications to previous specimen results on the basis of a single test improves laboratory turn-around-time and cost-efficiency. Another advantage of the chromogenic media is its ability to identify mixed cultures [11]. The identification of mixed cultures with C. glabrata or C. krusei may impact treatment decisions due to their intrinsic fluconazole resistance. The potential to identify mixed Candida cultures, the rapid presumptive species identification, and the improved turn-around-time of repeat positive specimens will significantly improve laboratory processes and ultimately patient care.

\section{ACKNOWLEDGEMENTS}

H. Adam is supported by a Clinical Microbiology Fellowship from the Ontario Ministry of Health and Long Term Care. The provision of the chromogenic media by Becton Dickinson Canada, Oakville, ON, Canada, Bio-Rad, Marnes-la-Coquette, France, and Oxoid Ltd., Nepean, ON, Canada is gratefully acknowledged.

\section{REFERENCES}

[1] Wisplinghoff H, Bischoff T, Tallent SM, Seifert H, Wenzel RP, Edmond MB. Nosocomial bloodstream infections in US hospitals: analysis of 24,179 cases from a prospective nationwide surveillance study. Clin Infect Dis 2004; 39: 309-17.

[2] Rangel-Frausto MS, Wiblin T, Blumberg HM, et al. National epidemiology of mycoses survey (NEMIS): variations in rates of bloodstream infections due to Candida species in seven surgical intensive care units and six neonatal intensive care units. Clin Infect Dis 1999; 29: 253-8.

[3] St-Germain G, Laverdiere M, Pelletier R, et al. Epidemiology and antifungal susceptibility of bloodstream Candida isolates in Quebec: Report on 453 cases between 2003 and 2005. Can J Infect Dis Med Microbiol 2008; 19: 55-62.

[4] Pappas PG, Rex JH, Sobel JD, et al. Guidelines for treatment of candidiasis. Clin Infect Dis 2004; 38: 161-89.

[5] Horvath LL, Hospenthal DR, Murray CK, Dooley DP. Direct isolation of Candida spp. from blood cultures on the chromogenic medium CHROMagar Candida. J Clin Microbiol 2003; 41: 262932.

[6] Hospenthal DR, Beckius ML, Floyd KL, Horvath LL, Murray CK. Presumptive identification of Candida species other than $C$. albicans, C. krusei, and C. tropicalis with the chromogenic medium CHROMagar Candida. Ann Clin Microbiol Antimicrob 2006; 5: $1-5$.

[7] Koehler AP, Chu KC, Houang ETS, Cheng AFB. Simple, reliable, and cost-effective yeast identification scheme for the clinical laboratory. J Clin Microbiol 1999; 37: 422-6.

[8] Murray CK, Beckius ML, Green JA, Hospenthal DR. Use of chromogenic medium in the isolation of yeasts from clinical specimens. J Med Microbiol 2005; 54: 981-5.

[9] Murray MP, Zinchuk R, Larone DH. CHROMagar Candida as the sole primary medium for isolation of yeasts and as a source medium for the rapid-assimilation-of-trehalose test. $\mathrm{J}$ Clin Microbiol 2005; 43: 1210-2.

[10] Willinger B, Hillowoth C, Selitsch B, Manafi M. Performance of candida ID, a new chromogenic medium for presumptive identification of Candida species, in comparison to CHROMagar Candida. J Clin Microbiol 2001; 39: 3793-5.

[11] Pfaller MA, Houston A, Coffmann S. Application of CHROMagar Candida for rapid screening of clinical specimens for Candida albicans, Candida tropicalis, Candida krusei, and Candida (Torulopsis) glabrata. J Clin Microbiol 1996; 34: 58-61.

[12] Biaxench MT, Taillandier A, Paugam A. Clinical and experimental evaluation of a new chromogenic medium (OCCA, Oxoid) for direct identification of Candida albicans, $C$. tropicalis, and $C$. krusei. Mycoses 2006; 49: 311-5. 
[13] Sendid B, Francois N, Standaert A, et al. Prospective evaluation of the new chromogenic medium CandiSelect 4 for differentiation and presumptive identification of the major pathogenic Candida speices. J Med Microbiol 2007; 56: 495-9.

[14] Chen YC, Eisner JD, Kattar MM, et al. Identification of medically important yeasts using PCR-based detection of DNA sequence polymorphisms in the internal transcribed spacer 2 region of the rRNA genes. J Clin Microbiol 2000; 38: 2302-10.

[15] NCCLS. Abbreviated Identification of Bacteria and Yeast; Approved Guideline. National Committee for Clinical Laboratory Standards. M35-A, 2000.

Received: October 02, 2009

Revised: February 06, 2010

Accepted: February 09, 2010

(C) Adam et al.; Licensee Bentham Open.

This is an open access article licensed under the terms of the Creative Commons Attribution Non-Commercial License (http://creativecommons.org/licenses/by$\mathrm{nc} / 3.0 /$ ), which permits unrestricted, non-commercial use, distribution and reproduction in any medium, provided the work is properly cited. 